\title{
New collocation scheme for solving fractional partial differential equations
}

\author{
Afshan Kanwal ${ }^{1,2}$ (D), Chang Phang*1 (D), Jian Rong Loh ${ }^{1,3}$ (ID \\ ${ }^{1}$ Department of Mathematics and Statistics, Universiti Tun Hussein Onn Malaysia, KM1, Jalan \\ Panchor, 84600 Pagoh, Johor, Malaysia \\ ${ }^{2}$ COMSATS University Islamabadad, Sahiwal Campus, COMSATS Road off G.T Road, Sahiwal, Pakistan \\ ${ }^{3}$ Foundation in Engineering, Faculty of Science and Engineering, University of Nottingham Malaysia, \\ Jalan Broga 43500 Semenyih, Selangor, Malaysia
}

\begin{abstract}
This article concerned about the numerical solution of time fractional partial differential equations (FPDEs). The proposed technique is using shifted Chebyshev-Gauss-Lobatto (CGL) collocation points in conjunction with an operational matrix of Caputo sense derivatives via Genocchi polynomials. The system of linear algebraic equations is obtained when the main equation along with the initial as well as boundary conditions is collocated by using shifted CGL collocation points. The main approach to this method is to transform the FPDEs to system of algebraic equations, hence, greatly simplify the numerical scheme. Comparison of the obtained results with the existing methods depicts that the suggested method is highly effect, more efficient and have less computational work. Some examples are given to illustrate the effectiveness and applicability of the proposed technique.
\end{abstract}

Mathematics Subject Classification (2010). 65M15, 65M99

Keywords. collocation method, shifted Chebyshev-Gauss-Lobatto, fractional partial differential equations, operational matrix

\section{Introduction}

The interest of researchers towards the study of fractional calculus and fractional partial differential equations (FPDEs) $[8,11,14,15,22-24]$ during the last few decades have been increased rapidly due to its significant role in modeling of many physical and engineering processes [8]. A number of fractional derivative applications and models can be found in chemical physics, viscoelasticity, anomalous diffusion, probability, astrophysics, optic and signal processing, electromagnetism and biology. When comparing with the integer order models, the memory and hereditary properties of different substances are well described by fractional-order models [22]. The FPDEs can be treated in three ways; time fractional and/or space fractional.

\footnotetext{
${ }^{*}$ Corresponding Author.

Email addresses: afshanumer21@gmail.com (A. Kanwal), pchang@uthm.edu.my (C. Phang), JianRong.Loh@nottingham.edu.my (J.R. Loh)

Received: 13.09.2018; Accepted: 19.08.2019
} 
In this research direction, the attempts for finding the solutions more accurately of various type of FPDEs including works in [4,5,26-29] . More specifically, in Turut and Güzel [21], they employed multivariate Padé approximation and variational iteration method to tackle FPDEs. Some FPDEs have been treated by Mohebbi et al. [12]. They have proposed compact difference scheme for the solution of FPDEs. Reutskiy [18] used the backward substitution method with Fourier series expansion for the solution of FPDEs and converted the given equation into a sequence of multi-term fractional ordinary differential equations (FODEs). Liu et al. [9] applied the fractional predictor-corrector method and the finite difference method to handle the multi-term time-fractional wave-diffusion equations. In [6], Ghandehari and Ranjbar solved the FPDEs by converting them into a nonlinear programming problem. The Sumudu transform method along with the Adomian polynomials have been proposed by Al-Khaled [1] for the solution of FPDEs.

On the other hand, in recent years, FPDEs have been also solved with the help of polynomials operational matrices for fractional integration and fractional differentiation as well. Mostly, the methods including spectral Tau method, collocation method and the Tau method could be used with the operational matrix method to solve the problem. Bhrawy and Zaky [2] investigated the time-fractional PDEs with variable coefficients. They applied spectral tau method by using fractional-order shifted Jacobi orthogonal function along with the operational matrix. Moreover, Patel et al. [16] used the collocation method which is based on 2D shifted Legendre polynomials for the solution of FPDEs. Yi et al. [25] applied the two-dimensional Block pulse operational matrix for FPDEs. Furthermore, Zhou and Xu [30] used the third kind of Chebyshev wavelets operational matrices of integration of fractional order to investigate the time-fractional convection diffusion equations with variable coefficients. The shifted Jacobi operational matrices along with the spectral tau method have been employed by Bhrawy and Zaky [3]. They solved some multi-term time-space FPDEs with Dirichlet boundary conditions. Saadatmandi et al. [19] deals with the variable coefficients based fractional convection-diffusion equations by using the combination of sinc functions and shifted Legendre polynomials in space and in time with the collocation method respectively.

Since the result for solving some FPDEs using operational matrix method based on orthogonal basis function is encouraging, the researches in this field may be can extended to other operational matrix method based on semi-orthogonal polynomials. We refer the readers to paper by Tohidi et al. [20] for some advantages of using semi-orthogonal polynomials like Bernoulli polynomials for approximating an arbitrary unknown function over some classical orthogonal polynomials. Other semi-orthogonal polynomials such as Appell polynomials may also had great potential to solve fractional calculus problems effectively. On top of that, we introduced operational matrix of derivative via Genocchi polynomials by Loh et al. [10] for the solution of fractional integro-differential equations, (FIDEs), Genocchi operational matrix of integration for solving fractional optimal control problems, (FOCPs) by Phang et al. [17] and pantograph equation [7]. Different than these recently developed schemes, here we solve the fractional partial differential equations (FPDEs) by using this Genocchi operational matrix of derivative in conjunction of shifted CGL. This shifted CGL together with Bernoulli operational matrix was succesfully applied to solve wave equations with Dirichlet boundary conditions and linear multidimensional diffusion equation by Zogheib [31] et al., but it is limited to the problem with integer derivative. Here, we hope that not only extend the Genocchi operational matrix to solve FPDEs, but also extend the use of shifted CGL collocation points in conjunction with semi-orthogonal polynomials to solve the FPDEs. 
The outline of the article is as follow. Some preliminaries related to fractional calculus, shifted CGL collocation points and Genocchi polynomials are discussed in Section 2. Section 3 is comprised of the description of the proposed technique. Section 4 consists of applications wherein solution of time FPDEs has been obtained via this new collocation scheme. Finally, we concluded our results in Section 5.

\section{Preliminaries}

Some of the basic definitions of fractional calculus are mentioned below:

Definition 1: The Riemann-Liouville $[11,14]$ (R-L) fractional derivative is defined as

where $n \in Z$.

$$
D_{t}^{\alpha} f(t)=\left\{\begin{array}{lc}
\frac{1}{\Gamma(n-\alpha)} \frac{d^{n}}{d t^{n}} \int_{0}^{t} \frac{f^{n}(\tau)}{(t-\tau)^{\alpha-n+1}} d \tau, & n-1<\alpha<n \\
\frac{d^{n}}{d t^{n}} f(t), & n=\alpha,
\end{array}\right.
$$

Definition 2: The Riemann-Liouville fractional integral operator $[11,14]$ of order $\alpha>0$, of a function $f \in C_{\mu}$, where $\mu \geq-1$, is defined as

$$
I^{\alpha} f(t)=\frac{1}{\Gamma(\alpha)} \int_{0}^{t}(t-\tau)^{\alpha-1} f(\tau) d \tau, \quad \alpha>0, \quad t>0 .
$$

Some Riemann-Liouville fractional integral properties are given as [11,14]

$$
\begin{aligned}
I^{0} f(t) & =f(t), \\
I^{\alpha} I^{\beta} f(t) & =I^{\alpha+\beta} f(t)=I^{\beta} I^{\alpha} f(t), \\
I^{\alpha} t^{\gamma} & =\frac{\Gamma(\gamma+1)}{\Gamma(\alpha+\gamma+1)} t^{\alpha+\gamma} .
\end{aligned}
$$

Definition 3: The fractional derivative in Caputo sense is defined as [11,14]

$$
D_{t}^{\alpha} f(t)=\left\{\begin{array}{lc}
\frac{1}{\Gamma_{n}^{n}(n-\alpha)} \int_{0}^{t} \frac{f^{n}(\tau)}{(t-\tau)^{\alpha-n+1}} d \tau, & n-1<\alpha<n, \\
\frac{d^{n}}{d t^{n}} f(t), & n=\alpha .
\end{array}\right.
$$

Some properties of Caputo sense fractional derivative are given as

$$
\begin{aligned}
D^{\alpha}(\lambda f(t)+\mu g(t)) & =\lambda D^{\alpha} f(t)+\mu D^{\alpha} g(t), \\
I^{\alpha} D_{t}^{\alpha} f(t) & =f(t)-\sum_{k=0}^{m-1} \frac{f^{(k)}\left(0^{+}\right)}{k !} t^{k}, t>0, \\
\frac{d^{n}}{d t^{n}}[\emptyset(t) f(t)] & =\sum_{k=0}^{n} \emptyset^{k}(t) f^{n-k}(t) .
\end{aligned}
$$

\subsection{Shifted Chebyshev-Gauss-Lobatto (CGL) nodes}

One of the main tool for this article is using shifted Chebyshev-Gauss-Lobatto (CGL) collocation points in conjunction with Genocchi operational matrix. Here, we briefly explain the concept. Suppose any function of two variables $v(x, t)$ can be approximated by using Genocchi polynomials, $G_{a}(x)$ and $G_{b}(t)$, where $a$ and $b$ denotes the degree of Genocchi polynomials,

$$
v_{M+1}(x, t) \approx \sum_{a=1}^{M+1} \sum_{b=1}^{M+1} c_{a b} G_{a}(x) G_{b}(t)
$$

in vector notation equation (2.6) can be written as,

$$
v(x, t)=\boldsymbol{G}^{T}(t) \boldsymbol{C}_{M+1 \times M+1} \boldsymbol{G}(x) .
$$


The Genocchi coefficient matrix $C$ which consist of the unique coefficients $c_{a b}$ follow as

$$
C^{T}=A^{-1} \mathcal{H}^{T} A^{-1}
$$

where, $\mathcal{H}=\int_{\mathbf{0}}^{\mathbf{1}} \int_{\mathbf{0}}^{\mathbf{1}} v(x, t) G_{a}(x) G_{b}(t) d x d t \quad$ and $\quad \boldsymbol{A}(x)=\int_{\mathbf{0}}^{\mathbf{1}} G_{a}(x) G_{a^{\prime}}(x) d x$, $\boldsymbol{A}(t)=\int_{\mathbf{0}}^{\mathbf{1}} G_{b}(t) G_{b^{\prime}}(t) d t$.

Let the basis functions are denoted by $\left\{\left\{G_{a}(x) G_{b}(t)\right\}_{a=1}^{M}\right\}_{b=1}^{M}$ associated with the points $\left\{x_{a}\right\}_{a=1}^{M}$ and $\left\{t_{b}\right\}_{b=1}^{M}$ correspondingly in the $x$ and $t$ directions. In this work, we are concerned with shifted CGL nodes. For this purpose, we let the extrema of $T_{M}(\eta)$ on $[-1,1]$ is given by $\eta=\cos \left(\frac{n \pi}{M}\right)$, where $T_{M}(\eta)$ be the Chebyshev polynomials of order $M$, we have the shifted CGL nodes given as $\eta_{0}^{(M)}, \ldots, \eta_{N}^{(M)}$.

Furthermore, we let Shifted CGL nodes based $\left\{\phi_{a}(\eta)\right\}_{a=1}^{M}$ Lagrange polynomials.

$$
\phi_{b}(\eta)=\prod_{a=0, a \neq b}^{M} \frac{\eta-\eta_{a}}{\eta_{b}-\eta_{a}}, \quad b=1, \ldots, M
$$

with the Kronecker property $\phi_{b}\left(\eta_{l}\right)=\delta_{b l}= \begin{cases}0, & b \neq l, \\ 1, & b=l .\end{cases}$

Also the formula named as Christoffel-Darboux given by the following relation can be used instead of equation (2.9)

$$
\phi_{b}(\eta)=\frac{(-1)^{b}\left(1-\eta^{2}\right)}{c_{a} N^{2}\left(\eta-\eta_{b}\right)} T_{M}^{\prime}(\eta), \text { where } c_{a}=\left\{\begin{array}{cc}
2, & a=0 \text { and } M \\
1, & 1 \leq a \leq M-1
\end{array}\right.
$$

Any defined function can be approximated by using the transformation from $[-1,1]$ to $[\zeta, \theta]$. For two variables, a function $w(x, t)$ may be approximated on the interval $[\zeta, \theta] \times[\zeta, \theta]$ and the nodes are created by the cosine distribution as

$$
\begin{aligned}
x_{a} & =\frac{\theta-\zeta}{2}\left(1-\cos \left(\frac{(a-1) \pi}{M}\right)\right)+\zeta, a=1,2, \ldots, M+1, \\
t_{b} & =\frac{\theta-\zeta}{2}\left(1-\cos \left(\frac{(b-1) \pi}{M}\right)\right)+\zeta, b=1,2, \ldots, M+1 .
\end{aligned}
$$

It is to be noted that the numerical solution becomes ambiguous near the domain boundaries with an increase in the order of field variable interpolation by the field nodes distributed uniformly. The problem can be tackle if the nodes are distributed densely near the boundaries and within the domain they are uniform. This can be done with the help of Chebyshev nodes. The shifted CGL nodes are used to solve any PDE because of the fact that it includes the domain boundaries as well. The Figures (1) and (2) represents the shifted CGL points for $M=4$ and $M=5$. 
- Initial collocation points

Boundary collocation points

$\triangle$ Interior collocation points

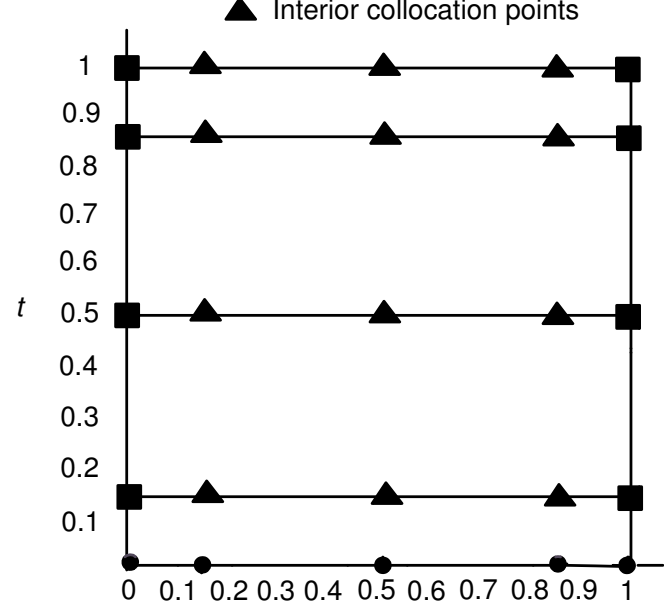

$x$

Figure 1. CGL collocations points for $M=4$.

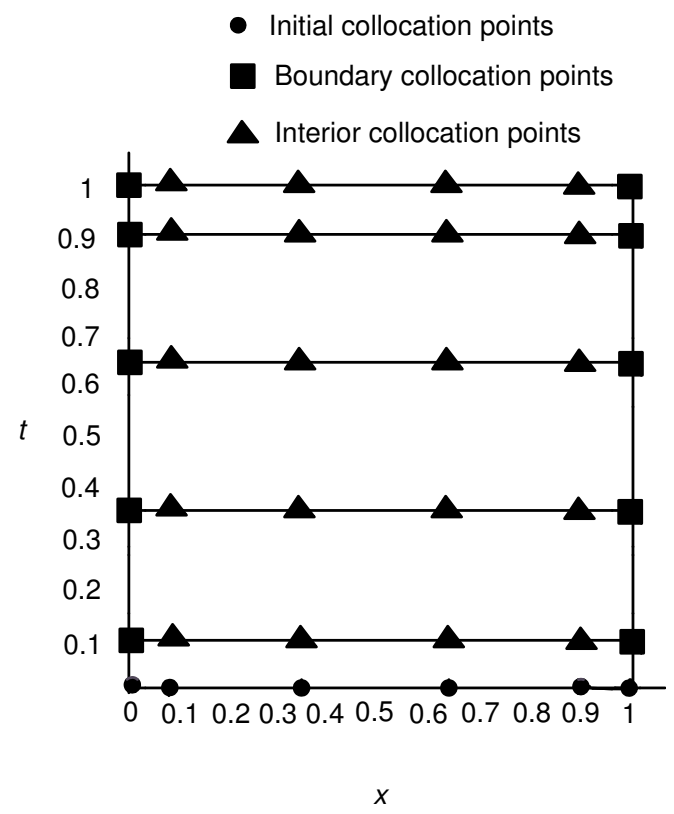

Figure 2. CGL collocations points for $M=5$.

\subsection{Two dimensional Genocchi polynomials}

The two-dimensional Genocchi polynomials are defined as a product function of two Genocchi polynomials.

$$
G_{a b}(x, t)=G_{a}(x) G_{b}(t) \quad a=1,2, \ldots, i, \quad b=1,2, \ldots, i .
$$

The expansion of any function $v(x, t)$ defined over the interval $[0,1] \times[0,1]$ in terms of Genocchi polynomials, can be written as

$$
v(x, t)=\sum_{a=1}^{\infty} \sum_{b=1}^{\infty} c_{a b} G_{a}(x) G_{b}(t)
$$


If the infinite series as given in equation (2.14) is truncated, then truncated form could be written as:

$$
v(x, t) \approx \sum_{a=1}^{i} \sum_{b=1}^{i} c_{a b} G_{a}(x) G_{b}(t)
$$

Theorem 2.1. Let $v(x, t) \in L^{2}[0,1]$ and $\left\{G_{k}(x), k=1,2, \ldots, S\right\}$ and $\left\{G_{l}(t), l=1,2, \ldots, H\right\}$ be two sets of Genocchi polynomials up to order $S$ and $H$, respectively.

Let $X=\operatorname{span}\left\{G_{1}(x), \ldots, G_{S}(x)\right\}$ and $X^{\prime}=\operatorname{span}\left\{G_{1}(t), \ldots, G_{H}(t)\right\}$. Since $X$ and $X^{\prime}$ are the finite dimensional subspaces of $L^{2}[0,1]$, then there exist $v^{*}(x, t)=X \times X^{\prime}$ is the best unique approximation in Genocchi polynomials such that $v(x, t)$ can be approximated by unique coefficients $c_{p q}$ as

$$
v(x, t)=\sum_{p=1}^{S} \sum_{q=1}^{H} c_{p q} G_{p}(x) G_{q}(t)=\boldsymbol{G}^{T}(x) \boldsymbol{C} \boldsymbol{G}(t) .
$$

where $\boldsymbol{C}$ the Genocchi coefficient matrix with the unique coefficients $c_{p q}$ given by $\boldsymbol{C}^{T}=$ $\boldsymbol{A}^{-1} \mathcal{H}^{\boldsymbol{T}} \boldsymbol{A}^{-1}$ and $\mathcal{H}=\int_{0}^{1} \int_{0}^{1} v(x, t) G_{p}(x) G_{q}(t) d x d t, \quad \boldsymbol{A}=\int_{0}^{1} G_{p}(t) G_{p^{\prime}}(t) d t$ or $\quad \boldsymbol{A}=$ $\int_{0}^{1} G_{q}(x) G_{q^{\prime}}(x) d x$.

Proof. Suppose we have

$$
v(x, t)=\sum_{p=1}^{S} \sum_{q=1}^{H} c_{p q} G_{p}(x) G_{q}(t),
$$

then multiply both sides with $G_{p^{\prime}}(x)$ and $G_{q^{\prime}}(t)$ and integrate, we have

$$
\int_{0}^{1} \int_{0}^{1} v(x, t) G_{p^{\prime}}(x) G_{q^{\prime}}(t) d x d t=\sum_{p=1}^{S} \sum_{q=1}^{H} c_{p q} \int_{0}^{1} G_{p}(x) G_{p^{\prime}}(x) d x \int_{0}^{1} G_{q}(t) G_{q^{\prime}}(t) d t .
$$

By letting, $v_{p^{\prime} q^{\prime}}(x, t)=\int_{0}^{1} \int_{0}^{1} v(x, t) G_{p^{\prime}}(x) G_{q^{\prime}}(t) d x d t$. Then we obtain

$$
v_{p^{\prime} q^{\prime}}(x, t)=\sum_{p=1}^{S} \sum_{q=1}^{H}\left(\int_{0}^{1} G_{p}(x) G_{p^{\prime}}(x) d x\right) c_{p q}\left(\int_{0}^{1} G_{q}(t) G_{q^{\prime}}(t) d t\right) .
$$

Both $v_{p^{\prime} q^{\prime}}(x, t)$ and $v(x, t)$ are similar. Hence we can write the equation $(2.17)$ as

$$
\mathcal{H}^{T}=A C^{T} A
$$

the coefficient matrix $C$ can be obtained by $C^{T}=A^{-1} \mathcal{H}^{T} A^{-1}$, where $A$ can be found from $\boldsymbol{A}=\int_{\mathbf{0}}^{\mathbf{1}} G_{p}(x) G_{p^{\prime}}(x) d x$ or $\boldsymbol{A}=\int_{\mathbf{0}}^{\mathbf{1}} G_{q}(t) G_{q^{\prime}}(t) d t$.

Lemma 2.2. Suppose that any function $u(x, t)$ be the sufficiently smooth function in domain $\omega$ and assume that it has derivative of any order bounded by a constant $F_{n}$ as follow,

$$
\left|\frac{\partial^{i+j-2} u(x, t)}{\partial^{i-1} x \partial^{j-1} t}\right| \leq F_{i-1, j-1}, i, j \in N .
$$

Also, let $u_{M}^{*}(x, t)$ be the approximation of $u(x, t)$ in terms of Genocchi polynomials, i.e.

$$
u(x, t) \approx u_{M}^{*}(x, t)=\sum_{i=1}^{M} \sum_{j=1}^{M} c_{i j} G_{i}(x) G_{j}(t),
$$

then we have the following maximum error in $L_{\infty}([0,1] \times[0,1])$ norm bounded by,

$$
\left\|u(x, t)-u_{M}^{*}(x, t)\right\|_{\infty} \leq \sum_{i=M+1}^{\infty} \sum_{j=M+1}^{\infty} \sum_{l=1}^{i} \sum_{m=1}^{j} \frac{F_{i-1, j-1}\left|g_{i-l}\right|\left|g_{j-m}\right|}{l ! m !(i-l) !(j-m) !}
$$


Remark: The equation (2.22) using Genocchi polynomials from 1 to $M$, hence we denote it as $u_{M}(x, t)$ while in $(2.6)$, we use $u_{M+1}(x, t)$ for represent Genocchi polynomials from 1 to $M+1$.

\section{Proof.}

$$
\begin{aligned}
\left\|u(x, t)-u_{M}^{*}(x, t)\right\|_{\infty} & =\max \left|u(x, t)-u_{M}^{*}(x, t)\right|, \\
& =\max \left|\sum_{i=M+1}^{\infty} \sum_{j=M+1}^{\infty} c_{i j} G_{i}(x) G_{j}(t)\right|, \\
& \leq \max \sum_{i=M+1}^{\infty} \sum_{j=M+1}^{\infty}\left|c_{i j} G_{i}(x) G_{j}(t)\right|, \\
& \leq \sum_{i=M+1}^{\infty} \sum_{j=M+1}^{\infty}\left|c_{i j}\right| \max \left|G_{i}(x)\right| G_{j}(t) \mid .
\end{aligned}
$$

where

$\left|c_{i j}\right|=\left|\frac{1}{4 i ! j !}\left[\frac{\partial^{i-1} \partial^{j-1}}{\partial^{i-1} x \partial^{j-1} t} u(1,1)+\frac{\partial^{i-1} \partial^{j-1}}{\partial^{i-1} x \partial^{j-1} t} u(1,0)+\frac{\partial^{i-1} \partial^{j-1}}{\partial^{i-1} x \partial^{j-1} t} u(0,1)+\frac{\partial^{i-1} \partial^{j-1}}{\partial^{i-1} x \partial^{j-1} t} u(0,0)\right]\right|$,

The terms $\left|c_{i j}\right|$ can be simplified as $\left|c_{i j}\right|=\left|\frac{1}{4 i ! j !}\left[4 F_{i-1, j-1}\right]\right|$, hence we obtain

$$
\left|c_{i j}\right|=\frac{F_{i-1, j-1}}{i ! j !} \text {. }
$$

Now, we have

$$
\max \left|G_{i}(x)\right|=\max \left|\sum_{l=1}^{i}\left(\begin{array}{c}
i \\
l
\end{array}\right) g_{i-l} x^{l}\right| \leq \sum_{l=1}^{i}\left(\begin{array}{c}
i \\
l
\end{array}\right)\left|g_{i-l}\right| .
$$

and similarly, we have

$$
\max \left|G_{j}(t)\right|=\max \left|\sum_{m=1}^{j}\left(\begin{array}{c}
j \\
m
\end{array}\right) g_{j-m} t^{m}\right| \leq \sum_{m=1}^{j}\left(\begin{array}{c}
j \\
m
\end{array}\right)\left|g_{j-m}\right| .
$$

By putting equations (2.25), (2.26) and (2.27) in equation (2.24), we have

$$
\begin{aligned}
\left\|u(x, t)-u_{M}^{*}(x, t)\right\|_{\infty} & \leq \sum_{i=M+1}^{\infty} \sum_{j=M+1}^{\infty} \sum_{l=1}^{i} \sum_{m=1}^{j} \frac{F_{i-1, j-1} \cdot i !\left|g_{i-l}\right| \cdot j !\left|g_{j-m}\right|}{i ! j ! l ! m !(i-l) !(j-m) !} \\
& \leq \sum_{i=M+1}^{\infty} \sum_{j=M+1}^{\infty} \sum_{l=1}^{i} \sum_{m=1}^{j} \frac{F_{i-1, j-1}\left|g_{i-l}\right|\left|g_{j-m}\right|}{l ! m !(i-l) !(j-m) !}
\end{aligned}
$$

Here, we give an example for approximate $u(x, t)=\sin (x) \sin (t)$ with using two dimensional Genocchi polynomials by using (2.22) and its error bound presented by above Lemma as in Table (1).

Table 1. Comparision of maximum error for approximate $u(x, t)=\sin (x) \sin (t)$ by using two dimensional Genocchi polynomials as in (2.22) and error bound by (2.24)

\begin{tabular}{ccc}
\hline M & Max error & Error bound \\
\hline 4 & $5.21823 \mathrm{E}-04$ & $5.79892 \mathrm{E}-02$ \\
5 & $4.70856 \mathrm{E}-05$ & $1.01132 \mathrm{E}-02$ \\
6 & $1.28722 \mathrm{E}-06$ & $5.54573 \mathrm{E}-04$ \\
7 & $1.14497 \mathrm{E}-06$ & $9.07230 \mathrm{E}-05$ \\
\hline
\end{tabular}


Here, we present the error analysis for the collocation scheme using shifted CGL nodes. Note that the first few Genoochi polynomials are $G_{1}(x)=1, G_{2}(x)=2 x-1$ and $G_{3}(x)=$ $3 x^{2}-3 x$. The Genocchi polynomials with $G_{i}(x)$ having $i-1$ highest power of $x$. On the other hand, using CGL points will give $M+1$ points. Hence, for the comparison of error for approximating function with Genocchi polynomials and interpolating with CGL points, we use $\omega_{N, N}^{*}(x, t)$ be its best approximation in terms of Genocchi polynomials, where degree $N$ is one degree higher than $M$. Assume that $\omega(x, t) \in \Xi$ be a sufficiently smooth function where $\Xi=[i, j] \times[k, l]$, then

$$
\begin{aligned}
\left\|\omega(x, t)-\omega_{N, N}^{*}(x, t)\right\|_{2} & \leq\left\|\omega(x, t)-\bar{\beth}_{M, M}(x, t)\right\|_{2} \\
& \leq \frac{1}{(M+1) ! 2^{2 r+1}}\left(\lambda_{1}+\lambda_{2}+\frac{\lambda_{3}}{(M+1) !\left(2^{2 r+1}\right)}\right),
\end{aligned}
$$

where $\lambda_{1}=\max _{(x, t) \in \bar{\beth}}\left|\frac{\partial^{M+1} \omega(x, t)}{\partial x^{M+1}}\right|, \lambda_{2}=\max _{(x, t) \in \bar{\beth}}\left|\frac{\partial^{M+1} \omega(x, t)}{\partial t^{M+1}}\right|$ and $\lambda_{3}=\max _{(x, t) \in \bar{\beth}}\left|\frac{\partial^{2 M+2} \omega(x, t)}{\partial x^{M+1} \partial t^{M+1}}\right|$.

Proof. The proof is following procedure in [13]. Let $\bar{\beth}_{M, M}(x, t)$ be the interpolating polynomials of the function $\omega(x, t)$ at points $\left(x_{a}, t_{b}\right)$ where $x_{a}$ and $t_{b}, a=0,1, \ldots, M, b=$ $0,1, \ldots, M$ are the shifted CGL nodes given by

$$
\begin{aligned}
x_{a} & =\frac{\theta-\zeta}{2}\left(1-\cos \left(\frac{a \pi}{M}\right)\right)+\zeta, a=0,1,2, \ldots, M, \\
t_{b} & =\frac{\theta-\zeta}{2}\left(1-\cos \left(\frac{b \pi}{M}\right)\right)+\zeta, b=0,1,2, \ldots, M,
\end{aligned}
$$

Then for any $(x, t) \in[i, j] \times[k, l]$, we can write

$$
\begin{array}{r}
\omega(x, t)-\bar{\beth}_{M, M}(x, t)=\frac{\partial^{M+1}}{\partial x^{M+1}} \frac{\omega(x, t)}{(M+1) !} \prod_{a=1}^{M+1}\left(x-x_{a}\right)+\frac{\partial^{M+1}}{\partial t^{M+1}} \\
\frac{\omega(x, t)}{(M+1) !} \prod_{b=1}^{M+1}\left(t-t_{b}\right)-\frac{\partial^{2 M+2}}{\partial x^{M+1} \partial t^{M+1}} \frac{\omega(x, t)}{(M+1) !^{2}} \prod_{a=1}^{M+1}\left(x-x_{a}\right) \prod_{b=1}^{M+1}\left(t-t_{b}\right),
\end{array}
$$

Thus we have

$$
\begin{array}{r}
\left|\omega(x, t)-\bar{\beth}_{M, M}(x, t)\right| \leq\left(\frac{\theta-\zeta}{2}\right)^{r+1} \frac{\lambda_{1}}{2^{r}(M+1) !}+ \\
\left(\frac{\theta-\zeta}{2}\right)^{r+1} \frac{\lambda_{2}}{2^{r}(M+1) !}+\left(\frac{\theta-\zeta}{2}\right)^{r+1}\left(\frac{\theta-\zeta}{2}\right)^{r+1} \frac{\lambda_{3}}{2^{r} .2^{r}(M+1) !^{2}}
\end{array}
$$

where

$$
\begin{aligned}
& \lambda_{1}=\max _{(x, t) \in \bar{\Xi}}\left|\frac{\partial^{M+1}}{\partial x^{M+1}} \omega(x, t)\right| \\
& \lambda_{2}=\max _{(x, t) \in \bar{\Xi}}\left|\frac{\partial^{M+1}}{\partial t^{M+1}} \omega(x, t)\right| \\
& \lambda_{3}=\max _{(x, t) \in \bar{\beth}}\left|\frac{\partial^{2 M+2}}{\partial x^{M+1} \partial t^{M+1}} \omega(x, t)\right|
\end{aligned}
$$


Since $\omega_{N, N}^{*}$ is the best approximation of $\omega(x, t)$, then finally by using equation (2.29), we get

$$
\begin{aligned}
& \left\|\omega(x, t)-\omega_{N, N}^{*}(x, t)\right\|_{2}^{2} \\
& \leq\left\|\omega(x, t)-\beth_{M, M}(x, t)\right\|_{2}^{2} \\
& =\int_{0}^{1} \int_{0}^{1}\left|\omega(x, t)-\bar{\beth}_{M, M}(x, t)\right|^{2} d x d t \\
& \leq \int_{0}^{1} \int_{0}^{1}\left(\left(\frac{\theta-\zeta}{2}\right)^{r+1} \frac{\lambda_{1}}{2^{r}(M+1) !}+\left(\frac{\theta-\zeta}{2}\right)^{r+1} \frac{\lambda_{2}}{2^{r}(M+1) !}\right. \\
& \left.\quad+\left(\frac{\theta-\zeta}{2}\right)^{2 r+2} \frac{\lambda_{3}}{2^{2 r}(M+1) !{ }^{2}}\right)^{2} d x d t . \\
& \leq\left(\left(\frac{\theta-\zeta}{2}\right)^{r+1} \frac{\lambda_{1}}{2^{r}(M+1) !}+\left(\frac{\theta-\zeta}{2}\right)^{r+1} \frac{\lambda_{2}}{2^{r}(M+1) !}+\left(\frac{\theta-\zeta}{2}\right)^{2 r+2} \frac{\lambda_{3}}{2^{2 r}(M+1) !^{2}}\right)^{2} .
\end{aligned}
$$

By taking the square root on both sides, we have

$$
\begin{aligned}
& \left\|\omega(x, t)-\omega_{N, N}^{*}(x, t)\right\|_{2} \\
& \quad \leq\left(\frac{\theta-\zeta}{2}\right)^{r+1} \frac{\lambda_{1}}{2^{r}(M+1) !}+\left(\frac{\theta-\zeta}{2}\right)^{r+1} \frac{\lambda_{2}}{2^{r}(M+1) !}+\left(\frac{\theta-\zeta}{2}\right)^{2 r+2} \frac{\lambda_{3}}{2^{2 r}(M+1) !^{2}} .
\end{aligned}
$$

Remark: The domain for the present problem is given by $\Xi=[0,1] \times[0,1]$, so for the error estimate the equation (2.36) becomes

$$
\left\|\omega(x, t)-\omega_{N, N}^{*}(x, t)\right\|_{2} \leq \frac{\lambda_{1}}{2^{2 r+1}(M+1) !}+\frac{\lambda_{2}}{2^{2 r+1}(M+1) !}+\frac{\lambda_{3}}{2^{4 r+2}(M+1) !^{2}}
$$

or can be written as

$$
\left\|\omega(x, t)-\omega_{N, N}^{*}(x, t)\right\|_{2} \leq \frac{1}{2^{2 r+1}(M+1) !}\left(\lambda_{1}+\lambda_{2}+\frac{\lambda_{3}}{2^{2 r+1}(M+1) !}\right),
$$

which concludes the proof.

Here, we give an example for error for approximate $u(x, t)=\sin (x) \sin (t)$ when $u(0.9,0.9)$ with using two dimensional Genocchi polynomials and compare with the error bound as in (2.29) as in Table (2). Note than since Genocchi Polynomials with degree $G_{N}(x)$ have $N-1$ power of $x$. Hence, we take one degree higher in $N$ to compare with same degree of Lagrange interpolation polynomials, $M$.

Table 2. Comparision of error for approximate $u(x, t)=\sin (x) \sin (t)$ when $u(0.9,0.9)$ with using two dimensional Genocchi polynomials and error bound by $(2.29)$

\begin{tabular}{cccc}
\hline $\mathbf{N}$ & Error & $\mathbf{M}$ & Error bound \\
\hline 3 & $1.02487 \mathrm{E}-03$ & 2 & $1.35593 \mathrm{E}-02$ \\
4 & $1.20530 \mathrm{E}-04$ & 3 & $9.22270 \mathrm{E}-04$ \\
5 & $1.75328 \mathrm{E}-05$ & 4 & $5.08672 \mathrm{E}-05$ \\
6 & $4.57160 \mathrm{E}-07$ & 5 & $1.92077 \mathrm{E}-06$ \\
\hline
\end{tabular}




\subsection{Genocchi operational matrix of derivative}

The matrix $U_{M \times M}^{\sigma}$ is the operational matrix of fractional derivative of order $\sigma$ in Caputo sense, we have

$$
\begin{aligned}
D_{t}^{\sigma} G(t) & =U_{M \times M}^{\sigma} G(t), \\
U_{M \times M}^{\sigma} & =\left[\begin{array}{cccc}
\theta_{11} & \theta_{12} & \cdots & \theta_{1 M} \\
\theta_{21} & \theta_{22} & \cdots & \theta_{2 M} \\
\vdots & \cdots & \cdots & \vdots \\
\theta_{M 1} & \theta_{M 2} & \cdots & \theta_{M M}
\end{array}\right], G(t)=\left[\begin{array}{c}
G_{1} \\
G_{2} \\
\vdots \\
G_{M}
\end{array}\right]
\end{aligned}
$$

Lemma 2.3. The formula for the fractional derivative of order $\sigma$ of Genocchi polynomials of order $k$ in Caputo sense is given as

$$
D_{t}^{\sigma} G_{i}(t)= \begin{cases}\sum_{l=\lceil\sigma\rceil \frac{i ! g_{i-l}}{(i-l) ! \Gamma(l-\sigma+1)}}^{i} t^{l-\sigma}, & n^{\prime}-1<\sigma \leq n^{\prime}, n^{\prime}=\lceil\sigma\rceil \in \mathbb{N}, i \geq \sigma \\ 0, & i<\sigma\end{cases}
$$

where $g_{i-l}$ is the Genocchi number.

Proof. See [10].

Lemma 2.4. The matrix $\aleph_{M}=\int_{0}^{1}\left(D_{t}^{\sigma} G(t)\right) * G^{T}(t) d t$ is given as $\aleph_{M}=\Phi_{i h}$, where we have

$$
\Phi_{i h}=\sum_{l=\lceil\sigma\rceil}^{i} \sum_{q=0}^{h} \frac{i !\left(\begin{array}{c}
h \\
q
\end{array}\right) g_{i-l} g_{h-q}}{(i-l) !(l+q-\sigma+1) \Gamma(l-\sigma+1)} .
$$

Proof. See [10].

Theorem 2.5. For the operational matrix of Genocchi polynomials in Caputo sense of fractional order $\sigma$ over the interval $[0,1]$, we let $G_{k}(x), k=1,2, \ldots, M$ be the set of Genocchi polynomials. We denote the operational matrix as $U_{M \times M}^{\sigma}$ which is given by

$$
U_{M \times M}^{\sigma}=\aleph_{M} A^{-1}
$$

Proof. See [10].

\section{Description of the proposed technique by using collocation method}

This section explain the procedure for the new collocation scheme, which is using shifted CGL collocation points in conjunction of Genocchi polynomials operational matrix method.

Consider the general fractional order partial differential equation as follow:

$$
\frac{\partial^{\alpha} v}{\partial t^{\alpha}}+\varpi_{1} \frac{\partial v}{\partial x}+\varpi_{2} \frac{\partial^{2} v}{\partial x^{2}}+v(x, t)=\omega(x, t), \quad(x, t) \in[\zeta, \theta] \times[\zeta, \theta],
$$

along with the following initial and boundary conditions

$$
\begin{gathered}
v(x, 0)=\varrho_{1}(x), \\
v(0, t)=\phi_{1}(t), v(1, t)=\phi_{2}(t) .
\end{gathered}
$$

where $\varpi_{1}$ and $\varpi_{2}$ are real parameters, $\omega$ is a continuous function and $\zeta=0$ and $\theta=1$. In matrix form, we approximate the solution $v(x, t)$ by means of Genocchi polynomials as

$$
v_{M+1}(x, t)=\sum_{a=1}^{M+1} \sum_{b=1}^{M+1} c_{a b} G_{a}(x) G_{b}(t)=\boldsymbol{G}^{T}(t) \boldsymbol{C}_{M+1 \times M+1} \boldsymbol{G}(x),
$$


where the Genocchi coefficient matrix $C_{M+1 \times M+1}$ is $M+1$ by $M+1$ matrix. The equations (3.1) to (3.3) can be approximate as

$$
\left.\begin{array}{rl}
\frac{\partial^{\alpha} v}{\partial t^{\alpha}} & =\boldsymbol{G}^{T}(t)\left(U_{M+1 \times M+1}^{\sigma}\right)^{T} \boldsymbol{C}_{M+1 \times M+1} \boldsymbol{G}(x), \\
\frac{\partial v}{\partial x} & =\boldsymbol{G}^{T}(t) \boldsymbol{C}_{M+1 \times M+1} U_{M+1 \times M+1}^{1} \boldsymbol{G}(x), \\
\frac{\partial^{2} v}{\partial x^{2}} & =\boldsymbol{G}^{T}(t) \boldsymbol{C}_{M+1 \times M+1} U_{M+1 \times M+1}^{2} \boldsymbol{G}(x) .
\end{array}\right\}
$$

Also

$$
\left.\begin{array}{rl}
v(x, 0) & =\boldsymbol{G}^{T}(0) \boldsymbol{C}_{M+1 \times M+1} \boldsymbol{G}(x), \\
v(0, t) & =\boldsymbol{G}^{T}(t) \boldsymbol{C}_{M+1 \times M+1} \boldsymbol{G}(0), \\
v(1, t) & =\boldsymbol{G}^{T}(t) \boldsymbol{C}_{M+1 \times M+1} \boldsymbol{G}(1) .
\end{array}\right\}
$$

By putting equations (3.5) and (3.6) in equations (3.1) to (3.3), we will get

$$
\begin{aligned}
& \boldsymbol{G}^{T}(t)\left(U_{M+1 \times M+1}^{\sigma}\right)^{T} \boldsymbol{C}_{M+1 \times M+1} \boldsymbol{G}(x)+\varpi_{1} \boldsymbol{G}^{T}(t) \boldsymbol{C}_{M+1 \times M+1} U_{M+1 \times M+1}^{1} \boldsymbol{G}(x) \\
& +\varpi_{2} \boldsymbol{G}^{T}(t) \boldsymbol{C}_{M+1 \times M+1} U_{M+1 \times M+1}^{2} \boldsymbol{G}(x)+\boldsymbol{G}^{T}(t) \boldsymbol{C}_{M+1 \times M+1} \boldsymbol{G}(x) \\
& =\omega(x, t) .
\end{aligned}
$$

and

$$
\left.\begin{array}{rl}
\boldsymbol{G}^{T}(0) \boldsymbol{C}_{M+1 \times M+1} \boldsymbol{G}(x) & =\varrho_{1}(x) \\
\boldsymbol{G}^{T}(t) \boldsymbol{C}_{M+1 \times M+1} \boldsymbol{G}(0) & =\phi_{1}(t) \\
\boldsymbol{G}^{T}(t) \boldsymbol{C}_{M+1 \times M+1} \boldsymbol{G}(1) & =\phi_{2}(t) .
\end{array}\right\}
$$

The shifted CGL collocation points are

$$
\begin{aligned}
x_{a} & =\frac{\theta-\zeta}{2}\left(1-\cos \left(\frac{(a-1) \pi}{M}\right)\right)+\zeta, & & a=1,2, \ldots, M+1, \\
t_{b} & =\frac{\theta-\zeta}{2}\left(1-\cos \left(\frac{(b-1) \pi}{M}\right)\right)+\zeta, & b & =1,2, \ldots, M+1,
\end{aligned}
$$

By using equations (3.7) and (3.8) with the collocation points, we can write the equation for equations (3.1) to (3.3) as

$$
\begin{aligned}
& \boldsymbol{G}^{T}\left(t_{b}\right)\left(U_{M+1 \times M+1}^{\sigma}\right)^{T} \boldsymbol{C}_{M+1 \times M+1} \boldsymbol{G}\left(x_{a}\right)+\varpi_{1} \boldsymbol{G}^{T}\left(t_{b}\right) \boldsymbol{C}_{M+1 \times M+1} U_{M+1 \times M+1}^{1} \boldsymbol{G}\left(x_{a}\right) \\
& +\varpi_{2} \boldsymbol{G}^{T}\left(t_{b}\right) \boldsymbol{C}_{M+1 \times M+1} U_{M+1 \times M+1}^{2} \boldsymbol{G}\left(x_{a}\right)+\boldsymbol{G}^{T}\left(t_{b}\right) \boldsymbol{C}_{M+1 \times M+1} \boldsymbol{G}\left(x_{a}\right) \\
& =\omega\left(x_{a}, t_{b}\right),
\end{aligned}
$$

where $\quad 2 \leq a \leq M, 2 \leq b \leq M+1$ and

$$
\left.\begin{array}{rlrl}
\boldsymbol{G}^{T}(0) \boldsymbol{C}_{M+1 \times M+1} \boldsymbol{G}\left(x_{a}\right) & =\varrho_{1}\left(x_{a}\right), & & 1 \leq a \leq M+1, \\
\boldsymbol{G}^{T}\left(t_{b}\right) \boldsymbol{C}_{M+1 \times M+1} \boldsymbol{G}(0) & =\phi_{1}\left(t_{b}\right), & & 2 \leq b \leq M+1, \\
\boldsymbol{G}^{T}\left(t_{b}\right) \boldsymbol{C}_{M+1 \times M+1} \boldsymbol{G}(1) & =\phi_{2}\left(t_{b}\right), & & 2 \leq b \leq M+1 .
\end{array}\right\}
$$

The system of linear algebraic equation can be obtained by combining the above equations. Hence, one can easily achieve the coefficients $c_{a b}$. Therefore, the approximate solution $v(x, t)$ can be calculated. For example, if $M=6$, the coefficent $\boldsymbol{C}_{M+1 \times M+1}$ is a matrix with dimension 7 by 7 . The equation in (3.10) will give 30 equations (i.e. 5 time 6 ), and equations in (3.11) will give 19 equations. (i.e. $7+6+6)$.

If there are initial condition $v_{t}(x, 0)=\varrho_{2}(x)$, we can have

$$
\boldsymbol{G}^{T}(0)\left(U_{M+1 \times M+1}^{1}\right)^{T} \boldsymbol{C}_{M+1 \times M+1} \boldsymbol{G}\left(x_{a}\right)=\varrho_{2}\left(x_{a}\right), \quad 1 \leq a \leq M+1 .
$$


In this case, some of the collocation points at interior point or/and boundary condition have to ignore in order to obtain system of linear algebraic equation with same number of equations and number of unknowns.

\section{Numerical results}

This section comprises of some numerical examples to show that the present technique is accurate for solving such FPDEs. The comparison results are also illustrated given below to verify the accuracy of present technique.

Problem 1. Consider the following linear time-fractional convection diffusion equation as in [30].

$$
\frac{\partial^{\alpha} u}{\partial t^{\alpha}}+x \frac{\partial u}{\partial x}-\frac{\partial^{2} u}{\partial x^{2}}=\frac{2 t^{2-\alpha}}{\Gamma(3-\alpha)}\left(x^{2}-x^{3}\right)+\left(1+t^{2}\right)\left(2 x^{2}-3 x^{3}+6 x-2\right),
$$

for $(x, t) \in[0,1] \times[0,1], \quad 0<\alpha<1$ where the initial and boundary conditions are given as:

$$
u(x, 0)=x^{2}-x^{3}, \quad u(0, t)=u(1, t)=0 .
$$

The exact solution is given by $u(x, t)=\left(1+t^{2}\right)\left(x^{2}-x^{3}\right)$. In Table (3), we compare our result by using the proposed technique with the result obtained by using Chebyshev wavelets method as shown in [30], which they use $k=2$ and $M=6$. Apart from that, we also show our result with different $\alpha$ at $M=3$ in Table (4).

Table 3. Comparison of the absolute errors for the method used in [30] with our proposed method for problem 1 for different values of $\alpha$ and $M=6$

\begin{tabular}{ccccc}
\hline \multirow{2}{*}{$(\mathbf{x}, \mathbf{t})$} & \multicolumn{3}{c}{$\boldsymbol{\alpha}=\mathbf{0 . 5}$} & \multicolumn{2}{c}{$\boldsymbol{\alpha = \mathbf { 0 . 7 }}$} \\
\cline { 2 - 5 } & {$[\mathbf{3 0}]$} & Proposed method & {$[30]$} & Proposed method \\
\hline$(0.1,0.1)$ & $6.3167 \mathrm{E}-06$ & $8.81552 \mathrm{E}-07$ & $1.0481 \mathrm{E}-05$ & $1.22521 \mathrm{E}-06$ \\
$(0.2,0.2)$ & $9.0144 \mathrm{E}-06$ & $8.70156 \mathrm{E}-07$ & $9.4357 \mathrm{E}-06$ & $1.62880 \mathrm{E}-06$ \\
$(0.3,0.3)$ & $1.0906 \mathrm{E}-05$ & $7.74359 \mathrm{E}-07$ & $1.0861 \mathrm{E}-05$ & $4.74839 \mathrm{E}-07$ \\
$(0.4,0.4)$ & $1.2217 \mathrm{E}-05$ & $2.02455 \mathrm{E}-06$ & $1.0863 \mathrm{E}-05$ & $2.70323 \mathrm{E}-06$ \\
$(0.5,0.5)$ & $1.2120 \mathrm{E}-05$ & $1.11899 \mathrm{E}-06$ & $9.8330 \mathrm{E}-06$ & $2.08419 \mathrm{E}-06$ \\
$(0.6,0.6)$ & $1.1359 \mathrm{E}-05$ & $1.12449 \mathrm{E}-06$ & $8.6775 \mathrm{E}-06$ & $1.00412 \mathrm{E}-06$ \\
$(0.7,0.7)$ & $9.6580 \mathrm{E}-06$ & $1.97722 \mathrm{E}-06$ & $7.0242 \mathrm{E}-06$ & $2.77912 \mathrm{E}-06$ \\
$(0.8,0.8)$ & $7.0716 \mathrm{E}-06$ & $3.40304 \mathrm{E}-07$ & $4.9338 \mathrm{E}-06$ & $9.02850 \mathrm{E}-07$ \\
$(0.9,0.9)$ & $3.7565 \mathrm{E}-06$ & $8.22466 \mathrm{E}-07$ & $2.5354 \mathrm{E}-06$ & $1.03032 \mathrm{E}-06$ \\
\hline
\end{tabular}

Table 4. Absolute errors of Problem 1 for different values of $\alpha$ and $M=3$

\begin{tabular}{ccccc}
\hline $\mathbf{( x , t )}$ & $\boldsymbol{\alpha}=\mathbf{0 . 1}$ & $\boldsymbol{\alpha}=\mathbf{0 . 3}$ & $\boldsymbol{\alpha}=\mathbf{0 . 7}$ & $\boldsymbol{\alpha = \mathbf { 0 . 9 }}$ \\
\hline$(0.1,0.1)$ & $1.05054 \mathrm{E}-07$ & $2.72011 \mathrm{E}-07$ & $2.40185 \mathrm{E}-07$ & $2.40185 \mathrm{E}-07$ \\
$(0.2,0.2)$ & $6.47738 \mathrm{E}-07$ & $2.00232 \mathrm{E}-06$ & $2.79383 \mathrm{E}-06$ & $2.79383 \mathrm{E}-06$ \\
$(0.3,0.3)$ & $1.72368 \mathrm{E}-06$ & $5.67480 \mathrm{E}-06$ & $8.83077 \mathrm{E}-06$ & $8.83077 \mathrm{E}-06$ \\
$(0.4,0.4)$ & $3.08438 \mathrm{E}-06$ & $1.05071 \mathrm{E}-05$ & $1.72204 \mathrm{E}-05$ & $1.72204 \mathrm{E}-05$ \\
$(0.5,0.5)$ & $4.20633 \mathrm{E}-06$ & $1.46759 \mathrm{E}-05$ & $2.48789 \mathrm{E}-05$ & $2.48789 \mathrm{E}-05$ \\
$(0.6,0.6)$ & $4.46786 \mathrm{E}-06$ & $1.59359 \mathrm{E}-05$ & $2.78249 \mathrm{E}-05$ & $2.78249 \mathrm{E}-05$ \\
$(0.7,0.7)$ & $3.43383 \mathrm{E}-06$ & $1.26330 \mathrm{E}-05$ & $2.29424 \mathrm{E}-05$ & $2.29424 \mathrm{E}-05$ \\
$(0.8,0.8)$ & $1.24802 \mathrm{E}-06$ & $5.11271 \mathrm{E}-06$ & $1.04523 \mathrm{E}-05$ & $1.04523 \mathrm{E}-05$ \\
$(0.9,0.9)$ & $8.66618 \mathrm{E}-07$ & $2.47919 \mathrm{E}-06$ & $2.90853 \mathrm{E}-06$ & $2.90853 \mathrm{E}-06$ \\
\hline
\end{tabular}


From the absolute error presented in Table (3), our method provide better result compare to those in [30].

Problem 2. Consider the following linear time-fractional diffusion-wave equation with damping as

$$
\frac{\partial^{\alpha} u}{\partial t^{\alpha}}+\frac{\partial u}{\partial t}=\frac{\partial^{2} u}{\partial x^{2}}+\rho(x, t),(x, t) \in[0,1] \times[0,1], \quad 1<\alpha \leq 2,
$$

where having the following initial and boundary conditions:

$$
u(x, 0)=0, \quad u_{t}(x, 0)=0, \quad u(0, t)=t^{3}, \quad u(1, t)=e t^{3} .
$$

The exact solution of the problem (2) is $u(x, t)=t^{3} e^{x}$ and $\rho(x, t)=3 t^{2} e^{x}-t^{3} e^{x}+\frac{6 e^{x} t^{3-\alpha}}{\Gamma(4-\alpha)}$. Using the suggested algorithm and for the different values of $M$ we obtained the numerical solutions. Table (5) shows the approximate solution of problem 2 for $\alpha=1.85$, while Table (6) shows the comparison in term of absolute error when equally space collocation is used compare to shifted CGL collocation, both with Genocchi operational matrix.

Table 5. Absolute errors of problem 2 for $\alpha=1.85$ and $M=4,6,8$

\begin{tabular}{cccc}
\hline$(\mathbf{x}, \mathbf{t})$ & $\mathbf{M}=\mathbf{4}$ & $\mathbf{M = 6}$ & $\mathbf{M = 8}$ \\
\hline$(0.1,0.1)$ & $2.93785 \mathrm{E}-03$ & $2.49737 \mathrm{E}-04$ & $2.79635 \mathrm{E}-04$ \\
$(0.2,0.2)$ & $1.38984 \mathrm{E}-02$ & $2.39629 \mathrm{E}-03$ & $8.60177 \mathrm{E}-03$ \\
$(0.3,0.3)$ & $2.57208 \mathrm{E}-02$ & $1.80089 \mathrm{E}-02$ & $2.40774 \mathrm{E}-02$ \\
$(0.4,0.4)$ & $3.01704 \mathrm{E}-02$ & $3.77154 \mathrm{E}-02$ & $3.76401 \mathrm{E}-02$ \\
$(0.5,0.5)$ & $2.50426 \mathrm{E}-02$ & $4.02594 \mathrm{E}-02$ & $3.86355 \mathrm{E}-02$ \\
$(0.6,0.6)$ & $1.39617 \mathrm{E}-02$ & $2.12280 \mathrm{E}-02$ & $2.39831 \mathrm{E}-02$ \\
$(0.7,0.7)$ & $2.59308 \mathrm{E}-03$ & $2.00676 \mathrm{E}-03$ & $3.76328 \mathrm{E}-03$ \\
$(0.8,0.8)$ & $5.18725 \mathrm{E}-03$ & $1.21360 \mathrm{E}-02$ & $9.01500 \mathrm{E}-03$ \\
$(0.9,0.9)$ & $7.80543 \mathrm{E}-03$ & $9.42216 \mathrm{E}-03$ & $9.02542 \mathrm{E}-03$ \\
\hline
\end{tabular}

Table 6. Comparison of the absolute errors for problem 2 with equally space/shifted CGL collocation points for $\alpha=1.85$ and $M=5$

\begin{tabular}{ccc}
\hline \multirow{2}{*}{$(\mathbf{x}, \mathbf{t})$} & \multicolumn{2}{c}{$\boldsymbol{M = 5}$} \\
\cline { 2 - 3 } & Equally Space & CGL \\
\hline$(0.2,0.2)$ & $8.14293 \mathrm{E}-03$ & $1.19147 \mathrm{E}-02$ \\
$(0.4,0.4)$ & $2.13116 \mathrm{E}-02$ & $3.53204 \mathrm{E}-02$ \\
$(0.6,0.6)$ & $2.81564 \mathrm{E}-02$ & $2.20159 \mathrm{E}-02$ \\
$(0.8,0.8)$ & $1.23640 \mathrm{E}-01$ & $7.86163 \mathrm{E}-03$ \\
\hline
\end{tabular}

From the absolute error presented in Table (5) and (6), our method provide better result compare to those in [30].

Problem 3. Consider the following linear time-fractional diffusion-wave equation with damping as [6]

$$
\frac{\partial^{\alpha} u}{\partial t^{\alpha}}+\frac{\partial u}{\partial t}-\frac{\partial^{2} u}{\partial x^{2}}=\frac{2 t^{2-\alpha}}{\Gamma(3-\alpha)}+2 x-2, \text { where }(x, t) \in[0,1] \times[0,1], \quad 0<\alpha \leq 1,
$$

where having the following initial and boundary conditions:

$$
u(x, 0)=x^{2}, \quad u(0, t)=t^{2}, \quad u(1, t)=1+t^{2} .
$$

This problem has the exact solution $u(x, t)=x^{2}+t^{2}$. Taking $M=4$ and using the proposed method described above to find the solution, we present the comparison of the 
absolute errors with already published results [6] in Tables (7) and (8) for $\alpha=0.5$. Table (9) shows the absolute error for $\alpha=0.5$, while Table (10) shows the comparison in term of absolute error when equally space collocation is used compare to shifted CGL collocation, both with Genocchi operational matrix.

Table 7. Comparison of the absolute errors of problem 3 with NLP by Ghandehari and Ranjbar [6] for $\alpha=0.5$ and $M=4$.

\begin{tabular}{ccccc}
\hline \multirow{2}{*}{$\mathbf{x}$} & \multicolumn{3}{c}{$\boldsymbol{t}=\mathbf{0 . 1}$} & $\boldsymbol{t}=\mathbf{0 . 5}$ \\
\cline { 2 - 5 } & {$[6]$} & Proposed method & {$[6]$} & Proposed method \\
\hline 0.1 & $3.8678 \mathrm{E}-04$ & $1.7490 \mathrm{E}-05$ & $3.7450 \mathrm{E}-04$ & $1.3056 \mathrm{E}-05$ \\
0.2 & $6.9640 \mathrm{E}-04$ & $3.1345 \mathrm{E}-05$ & $6.5970 \mathrm{E}-04$ & $2.4353 \mathrm{E}-05$ \\
0.3 & $9.3370 \mathrm{E}-04$ & $4.1768 \mathrm{E}-05$ & $8.5790 \mathrm{E}-04$ & $3.3392 \mathrm{E}-05$ \\
0.4 & $1.1000 \mathrm{E}-03$ & $4.8791 \mathrm{E}-05$ & $9.8740 \mathrm{E}-04$ & $3.9716 \mathrm{E}-05$ \\
0.5 & $1.2000 \mathrm{E}-03$ & $5.2278 \mathrm{E}-05$ & $1.0000 \mathrm{E}-03$ & $4.2906 \mathrm{E}-05$ \\
0.6 & $1.2000 \mathrm{E}-03$ & $5.1923 \mathrm{E}-05$ & $8.9350 \mathrm{E}-04$ & $4.2582 \mathrm{E}-05$ \\
0.7 & $1.1000 \mathrm{E}-03$ & $4.7248 \mathrm{E}-05$ & $6.4050 \mathrm{E}-04$ & $3.8409 \mathrm{E}-05$ \\
0.8 & $9.9630 \mathrm{E}-04$ & $3.7608 \mathrm{E}-05$ & $2.3080 \mathrm{E}-04$ & $3.0086 \mathrm{E}-05$ \\
0.9 & $7.4310 \mathrm{E}-04$ & $2.2187 \mathrm{E}-05$ & $1.8500 \mathrm{E}-04$ & $1.7355 \mathrm{E}-05$ \\
\hline
\end{tabular}

Table 8. Comparison of the absolute errors of problem 3 with NLP by Ghandehari and Ranjbar [6] for $\alpha=0.5$ and $M=4$.

\begin{tabular}{ccc}
\hline \multirow{2}{*}{$\mathbf{x}$} & \multicolumn{2}{c}{$\boldsymbol{t}=\mathbf{1}$} \\
\cline { 2 - 3 } & {$[6]$} & Proposed method \\
\hline 0.1 & $3.1600 \mathrm{E}-04$ & $4.1324 \mathrm{E}-05$ \\
0.2 & $5.2900 \mathrm{E}-04$ & $7.3127 \mathrm{E}-05$ \\
0.3 & $6.4100 \mathrm{E}-04$ & $9.6523 \mathrm{E}-05$ \\
0.4 & $6.7800 \mathrm{E}-04$ & $1.1206 \mathrm{E}-04$ \\
0.5 & $6.2700 \mathrm{E}-03$ & $1.1973 \mathrm{E}-04$ \\
0.6 & $4.6800 \mathrm{E}-04$ & $1.1895 \mathrm{E}-04$ \\
0.7 & $2.0100 \mathrm{E}-04$ & $1.0857 \mathrm{E}-04$ \\
0.8 & $1.9700 \mathrm{E}-04$ & $8.6901 \mathrm{E}-05$ \\
0.9 & $4.0000 \mathrm{E}-05$ & $5.1654 \mathrm{E}-05$ \\
\hline
\end{tabular}

Table 9. Absolute errors of problem 3 for $\alpha=0.5$ and different values of $t$ and $M$.

\begin{tabular}{cccc}
\hline $\mathbf{t}$ & $\mathbf{M = 5}$ & $\mathbf{M = 6}$ & $\mathbf{M = 8}$ \\
\hline 0.1 & $1.6303 \mathrm{E}-05$ & $1.1452 \mathrm{E}-05$ & $2.8151 \mathrm{E}-06$ \\
0.5 & $1.1943 \mathrm{E}-05$ & $3.1877 \mathrm{E}-06$ & $1.1780 \mathrm{E}-06$ \\
1 & $1.7873 \mathrm{E}-05$ & $8.9557 \mathrm{E}-06$ & $2.8867 \mathrm{E}-06$ \\
\hline
\end{tabular}


Table 10. Comparison of the absolute errors for problem 3 with Equally space/shifted CGL collocation points for $\alpha=0.5, M=4$ and different values of $t$.

\begin{tabular}{ccccc}
\hline \multirow{2}{*}{$(\mathbf{x}, \mathbf{t})$} & \multicolumn{2}{c}{$\boldsymbol{t}=\mathbf{0 . 1}$} & \multicolumn{2}{c}{$\boldsymbol{t}=\mathbf{0 . 5}$} \\
\cline { 2 - 5 } & Equally Space & CGL & Equally Space & CGL \\
\hline$(0.2,0.2)$ & $0.507858 \mathrm{E}-04$ & $3.13452 \mathrm{E}-05$ & $0.125709 \mathrm{E}-04$ & $0.243533 \mathrm{E}-04$ \\
$(0.4,0.4)$ & $0.800191 \mathrm{E}-04$ & $4.87915 \mathrm{E}-05$ & $1.90050 \mathrm{E}-05$ & $0.397165 \mathrm{E}-04$ \\
$(0.6,0.6)$ & $8.51427 \mathrm{E}-05$ & $5.19230 \mathrm{E}-05$ & $1.99898 \mathrm{E}-05$ & $0.425829 \mathrm{E}-04$ \\
$(0.8,0.8)$ & $0.610328 \mathrm{E}-04$ & $3.76082 \mathrm{E}-05$ & $1.45404 \mathrm{E}-05$ & $3.00861 \mathrm{E}-05$ \\
\hline
\end{tabular}

Problem 4. Let us now consider the linear time-fractional convection diffusion equation is as follow [19]

$$
\frac{\partial^{\alpha} u}{\partial t^{\alpha}}+x \frac{\partial u}{\partial x}+\frac{\partial^{2} u}{\partial x^{2}}=2 t^{\alpha}+2 x^{2}+2, \quad(x, t) \in[0,1] \times[0,1], \quad 0<\alpha \leq 1,
$$

with initial and boundary conditions:

$$
u(x, 0)=x^{2}, \quad u(0, t)=\frac{2 \Gamma(\alpha+1)}{\Gamma(2 \alpha+1)} t^{2 \alpha}, \quad u(1, t)=1+\frac{2 \Gamma(\alpha+1)}{\Gamma(2 \alpha+1)} t^{2 \alpha} .
$$

The exact solution of the problem (4) is $u(x, t)=x^{2}+\frac{2 \Gamma(\alpha+1)}{\Gamma(2 \alpha+1)} t^{2 \alpha}$. Proceeding as before and taking different values of $M$ we get the approximate solution. Table (11) and Table (12) illustrate the comparison of the absolute errors with existing results (Table 4, Example 3) in [19] for $\alpha=0.5$, where the writers used Sinc-Legendre collocation method for $m=15$ and $m=25$. Also Table (13) depicts the comparison of the absolute errors with result in [1] where Sumudu decomposition method is applied for $\alpha=0.75$ and $\alpha=0.95$.

Table 11. Comparison of the obtained solutions of problem 4 with existing results by Saadatmandi and Dehghan [19] for $\alpha=0.5, t=0.5$ and $M=4$.

\begin{tabular}{cccc}
\hline \multirow{2}{*}{$\mathbf{x}$} & $\mathbf{m}=\mathbf{1 5}$ & $\mathbf{m}=\mathbf{2 5}$ & $\mathbf{M}=\mathbf{4}$ \\
\cline { 2 - 4 } & {$[\mathbf{1 9}]$} & {$[\mathbf{1 9}]$} & Proposed method \\
\hline 0.1 & $6.994 \mathrm{E}-05$ & $6.462 \mathrm{E}-06$ & $1.42760 \mathrm{E}-04$ \\
0.2 & $1.721 \mathrm{E}-04$ & $1.578 \mathrm{E}-05$ & $2.42446 \mathrm{E}-04$ \\
0.3 & $2.472 \mathrm{E}-04$ & $2.272 \mathrm{E}-05$ & $3.06440 \mathrm{E}-04$ \\
0.4 & $2.912 \mathrm{E}-04$ & $2.674 \mathrm{E}-05$ & $3.40341 \mathrm{E}-04$ \\
0.5 & $3.004 \mathrm{E}-04$ & $2.759 \mathrm{E}-05$ & $3.47955 \mathrm{E}-04$ \\
\hline
\end{tabular}

Table 12. Absolute errors of problem 4 for $\alpha=0.5, t=0.5$ and different values of $M$.

\begin{tabular}{ccc}
\hline \multirow{2}{*}{$\mathbf{x}$} & $\mathbf{M = 6}$ & $\mathbf{M = 8}$ \\
\cline { 2 - 3 } & Proposed method & Proposed method \\
\hline 0.1 & $4.62306 \mathrm{E}-05$ & $6.93784 \mathrm{E}-06$ \\
0.2 & $7.44193 \mathrm{E}-05$ & $2.10061 \mathrm{E}-05$ \\
0.3 & $9.02312 \mathrm{E}-05$ & $3.51184 \mathrm{E}-05$ \\
0.4 & $9.79210 \mathrm{E}-05$ & $4.37771 \mathrm{E}-05$ \\
0.5 & $1.00046 \mathrm{E}-04$ & $4.40540 \mathrm{E}-05$ \\
0.6 & $9.73594 \mathrm{E}-05$ & $3.60376 \mathrm{E}-05$ \\
0.7 & $8.88893 \mathrm{E}-05$ & $2.25994 \mathrm{E}-05$ \\
0.8 & $7.22028 \mathrm{E}-05$ & $8.54433 \mathrm{E}-06$ \\
0.9 & $4.38510 \mathrm{E}-05$ & $6.24458 \mathrm{E}-07$ \\
\hline
\end{tabular}


Table 13. Comparison of the obtained solutions of problem 4 with existing results by Al-Khaled [1] for different values of $\alpha$.

\begin{tabular}{ccccccc}
\hline \multirow{2}{*}{$\mathbf{t}$} & $\mathbf{x}$ & {$[\mathbf{1}]$} & Proposed method & Exact & {$[1]$} & $\boldsymbol{\alpha}=\mathbf{0 . 9 5}$ \\
\cline { 3 - 7 } & & 0.163679 & 0.164034 & 0.16367554 & 0.090389 & 0.090417 \\
\hline 0.2 & 0.2 & 0.150 osed method \\
& 0.4 & 0.283679 & 0.284154 & 0.28367554 & 0.210389 & 0.210426 \\
& 0.7 & 0.61368 & 0.614087 & 0.61367554 & 0.540421 & 0.540421 \\
0.5 & 0.2 & 0.530488 & 0.528245 & 0.52887053 & 0.327330 & 0.327330 \\
& 0.4 & 0.650675 & 0.648210 & 0.64887053 & 0.447341 & 0.447341 \\
& 0.7 & 0.981188 & 0.978227 & 0.97887053 & 0.777334 & 0.777334 \\
0.8 & 0.2 & 1.06802 & 1.029419 & 1.02940439 & 0.742482 & 0.742482 \\
& 0.4 & 1.19247 & 1.149374 & 1.14940439 & 0.862833 & 0.862833 \\
& 0.7 & 1.53473 & 1.479389 & 1.47940439 & 1.192619 & 1.192619 \\
\hline
\end{tabular}

From Tables (11)- (13), it is to be noted that the proposed technique gives better results as compare to the existing [19] and [1].

For the solution obtained with different $\alpha$, we show it as in Figure (3) and (4).

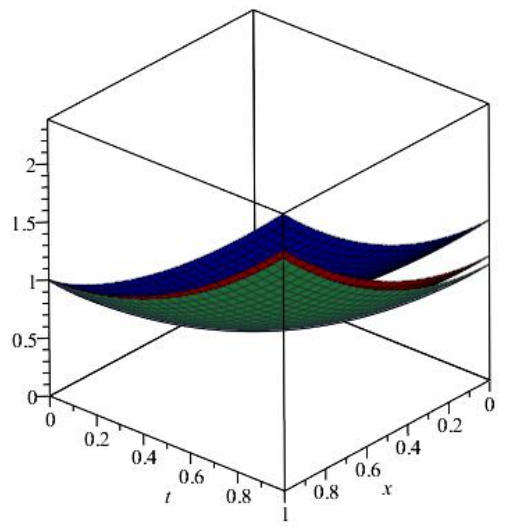

Figure 3. Solution $u(x, t)$ for different $\alpha$, which $\alpha=0.75$ : Green, $\alpha=0.95$ : Blue, $\alpha=1:$ Red

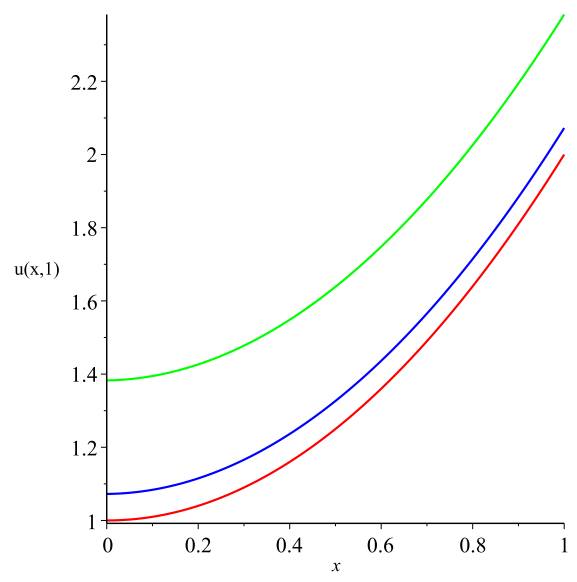

Figure 4. Solution $u(x, 1)$ for different $\alpha$, which $\alpha=0.75$ : Green, $\alpha=0.95$ : Blue, $\alpha=1: \operatorname{Red}$ 
Problem 5. Consider the following nonlinear FPDE as follow

$$
\frac{\partial^{\alpha} u}{\partial t^{0.5}}+\frac{\partial u}{\partial t}+\sin (u)=\frac{8}{3} \frac{t^{3 / 2} x}{\sqrt{\pi}}+\sin \left(x t^{2}\right),(x, t) \in[0,1] \times[0,1],
$$

where having the following initial and boundary conditions:

$$
u(x, 0)=0, \quad u(0, t)=0, \quad u(1, t)=t .
$$

The exact solution of the problem $(5)$ is $u(x, t)=x t^{2}$. Using the suggested algorithm and for the different values of $M$ we obtained the numerical solutions. Table (14) shows the approximate solution of problem 5 .

Table 14. Absolute error for the solutions of problem 5

\begin{tabular}{ccc}
\hline \multirow{2}{*}{$(\mathbf{x}, \mathbf{t})$} & \multicolumn{2}{c}{$\boldsymbol{M}$} \\
\cline { 2 - 3 } & $\boldsymbol{M}=\mathbf{3}$ & $\boldsymbol{M}=\mathbf{6}$ \\
\hline$(0.1,0.1)$ & $8.64223 \mathrm{E}-04$ & $7.61729 \mathrm{E}-04$ \\
$(0.1,0.5)$ & $1.02828 \mathrm{E}-02$ & $9.98584 \mathrm{E}-03$ \\
$(0.1,0.8)$ & $2.19334 \mathrm{E}-02$ & $2.13345 \mathrm{E}-02$ \\
$(0.5,0.1)$ & $1.20605 \mathrm{E}-05$ & $3.35229 \mathrm{E}-05$ \\
$(0.5,0.5)$ & $1.93707 \mathrm{E}-04$ & $7.86163 \mathrm{E}-05$ \\
$(0.5,0.8)$ & $2.78549 \mathrm{E}-04$ & $1.73250 \mathrm{E}-04$ \\
\hline
\end{tabular}

From the result in Table (14), the propose scheme is also applicable to solve the nonlinear FPDE.

\section{Conclusion}

In this paper, we used shifted Chebyshev-Gauss-Lobatto collocation points in conjunction with Genocchi polynomials operational matrix of differentiation in Caputo sense to solve fractional partial differential equations. The system of linear algebraic equations is obtained when the main equation along with the initial as well as boundary conditions is collocated by using shifted CGL collocation points, hence, greatly simplify the numerical scheme. Comparison tables are given to show the accuracy of present technique. It is worth noting that our propose method gives better results when compared with existing work.

Acknowledgment. This research is supported by the Universiti Tun Hussein Onn Malaysia under GPPS Vot U304 and Ministry of Education Malaysia under the Fundamental Research Grant Scheme (FRGS) Vot K072.

\section{References}

[1] K. Al-Khaled, Numerical solution of time-fractional partial differential equations using Sumudu decomposition method, Rom. J. Phys. 60 (1-2), 99-110, 2015.

[2] A.H. Bhrawy and M.A. Zaky, Fractional-order Jacobi Tau method for a class of timefractional PDEs with variable coefficients, Math. Methods Appl. Sci. 16 (4), 490-498, 2015.

[3] A.H. Bhrawy and M.A. Zaky, A method based on the Jacobi Tau approximation for solving multi-term time-space fractional partial differential equations, J. Comput. Phys. 281, 876-895, 2015.

[4] A.H. Bhrawy and M.A. Zaky, An improved collocation method for multi-dimensional space-time variable-order fractional Schrodinger equations, Appl. Numer. Math. 111, 197-218, 2017. 
[5] A.H. Bhrawy and M.A. Zaky, Numerical simulation of multi-dimensional distributedorder generalized Schrodinger equations, Nonlinear Dyn. 89 (2), 1415-1432, 2017.

[6] M.A.M. Ghandehari and M. Ranjbar, A numerical method for solving a fractional partial differential equation through converting it into an NLP problem, Comput. Math. Appl. 65 (7), 975-982, 2013.

[7] A. Isah, C. Phang and P. Phang, Collocation method based on Genocchi operational matrix for solving generalized fractional Pantograph equations, Int. J. Differ. Equ. 2017, 2017.

[8] H. Jiang, F. Liu, I. Turner and K. Burrage, Analytical solutions for the multi-term time-fractional diffusion- wave/diffusion equations in a finite domain, Comput. Math. Appl. 64 (10), 3377-3388, 2012.

[9] F. Liu, M. Meerschaert, R. McGough, P. Zhuang and Q. Liu, Numerical methods for solving the multi-term time-fractional wave-diffusion equation, Fract. Calc. Appl. Anal. 16 (1), 9-25, 2013.

[10] J.R. Loh, C. Phang and A. Isah, New operational matrix via Genocchi polynomials for solving Fredholm-Volterra fractional integro-differential equations (FIDEs), Adv. Math. Phys. 2017, 2017.

[11] K.S. Miller and B. Ross, An introduction to the fractional calculus and fractional differential equations, Wiley-Interscience, 1993.

[12] A. Mohebbi, M. Abbaszadeh and M. Dehghan, High-order difference scheme for the solution of linear time fractional Klein-Gordon equations, Numer. Methods Partial Diff. Equ. 30 (4), 1234-1253, 2014.

[13] S. Nemati and Y. Ordokhani, Legendre expansion methods for the numerical solution of nonlinear $2 D$ Fredholm integral equations of the second kind, J. Appl. Math. Informatics, 31 (5-6), 609-621. 2013.

[14] K. Oldham and J. Spanier, The fractional calculus theory and applications of differentiation and integration to arbitrary order, Elsevier, 1974.

[15] N. Ozdemir and M. Yavuz, Numerical solution of fractional Black-Scholes equation by using the multivariate Pade approximation, Acta Phys. Pol. A, 132 (3), 1050-1053, 2017.

[16] V.K. Patel, S. Somveer and V.K. Singh, Two-dimensional shifted Legendre polynomial collocation method for electromagnetic waves in dielectric media via almost operational matrices, Math. Methods Appl. Sci., 2017.

[17] C. Phang, N.F. Ismail, A. Isah and J.R. Loh, A new efficient numerical scheme for solving fractional optimal control problems via a Genocchi operational matrix of integration, J. Vib. Control 24 (14), 3036-3048, 2018.

[18] S.Y. Reutskiy, A new semi-analytical collocation method for solving multi-term fractional partial differential equations with time variable coefficients, Appl. Math. Model. 45, 238-254, 2017.

[19] A. Saadatmandi, M. Dehghan and M.R. Azizi, The Sinc-Legendre collocation method for a class of fractional convection-diffusion equations with variable coefficients, Commun. Nonlinear Sci. Numer. Simul. 17 (11), 4125-4136, 2012.

[20] E. Tohidi, A.H. Bhrawy and K. Erfani, A collocation method based on Bernoulli operational matrix for numerical solution of generalized pantograph equation, Appl. Math. Model. 37 (6), 4283-4294, 2013.

[21] V. Turut and N. Güzel, On solving partial differential equations of fractional order by using the variational iteration method and multivariate Padé approximations, Eur. J. Pure Appl. Math. 6 (2), 147-171, 2013.

[22] V.A. Vyawahare and P.S.V. Nataraj, Fractional-order modeling of neutron transport in a nuclear reactor, Appl. Math. Model. 37 (23), 9747-9767, 2013.

[23] M. Yavuz and N. Ozdemir, A different approach to the European option pricing model with new fractional operator, Math. Model. Nat. Pheno. 13 (1), 12, 2018. 
[24] M. Yavuz, N. Ozdemir, and H.M. Baskonus, Solutions of partial differential equations using the fractional operator involving Mittag-Leffler kernel, Eur. Phys. J. Plus, 133 (6), 215, 2018.

[25] M. Yi, J. Huang and J. Wei, Block pulse operational matrix method for solving fractional partial differential equation, Appl. Math. Comput. 221, 121-131, 2013.

[26] M.A. Zaky, An improved Tau method for the multi-dimensional fractional RayleighStokes problem for a heated generalized second grade fluid, Comput. Math. Appl. 75 (7), 2243-2258, 2018.

[27] M.A. Zaky, A Legendre spectral quadrature tau method for the multi-term timefractional diffusion equations, Computat. Appl. Math. 37 (3), 3525-3538, 2018.

[28] M.A. Zaky, Recovery of high order accuracy in Jacobi spectral collocation methods for fractional terminal value problems with non-smooth solutions, J. Comput. Appl. Math. 357, 103-122, 2019.

[29] M.A. Zaky, E.H. Doha and J.T. Machado, A spectral framework for fractional variational problems based on fractional Jacobi functions, Appl. Numer. Math. 132, 51-72, 2018.

[30] F. Zhou and X. Xu, The third kind Chebyshev wavelets collocation method for solving the time-fractional convection diffusion equations with variable coefficients, Appl. Math. Comput. 280, 11-29, 2016.

[31] B. Zogheib, E. Tohidi and S. Shateyi, Bernoulli collocation method for solving linear multidimensional diffusion and wave equations with Dirichlet boundary conditions, Adv. Math. Phys. 2017, 2017. 